\title{
Optimized Design and Simulation of Resonant Harmonic Filter
}

\author{
Tong Xiangqian Shen Wei Deng Yaping
}

School of Electrical Engineering, Xi'an University of Technology, Xi'an, 710048, China

\begin{abstract}
A new way to obtain the optimal tuning frequency of a single-tuned harmonic filter is presented, so as to control harmonic current to the allowable value with a minimum or given capacity of the filter. A PC-based filter design procedure accompanied by a simulation code to verify the effectiveness of the filter was developed based on MATLAB. An industrial system was selected to demonstrate the application of the proposed procedure.
\end{abstract}

Keywords: Harmonic, single-tuned filter, detuning, filter design

\section{Introduction}

Nonlinear loads cause serious harmonic distortion in power system[1], and singletuned filter (STF) is commonly used for both harmonic suppression and reactive power compensation. But filter detuning, caused by variation of system frequency and filter elements, significantly decrease the filter's efficiency and may cause overload in filter. Therefore, it is recommended that the tuning frequency of STF is partial from and below the suppressed harmonic frequency[2-3].

This paper presents a new design method for a STF by optimizing the tuning frequency, intended to control harmonic distortion within the IEEE limits with a minimum or given capacity predetermined by reactive power compensation. A computer-aided design procedure for STF and an associated simulation code for analysis of industrial power system with harmonic filters are illustrated.

\section{Harmonic Model of Power System}

An industrial power system with nonlinear loads and STF is shown in Fig.1, and Fig.2 shows its equivalent circuit for harmonic analysis and filter design. Supply system is represented as an ideal voltage source in series with an inductance denoted by $L_{s}$. The nonlinear load is represented as multiple harmonic current sources denoted by $\mathrm{I}_{\mathrm{h}}$, and its value is usually derived from actual measurement. The linear load and the power factor correction capacitor with a series reactor, is represented by load impedance. The STF is equivalent to a RLC branch.

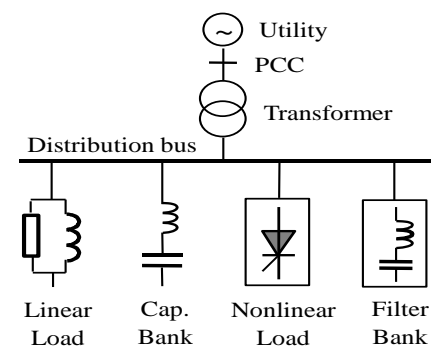

Fig.1 Power system with harmonic load and filter

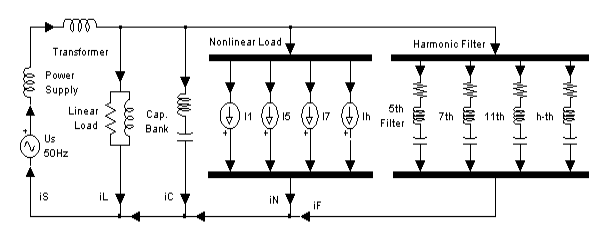

Fig. 2 Equivalent circuit for filter design 
For a specific $h^{\text {th }}$ harmonic, the effect of linear loads, including the capacitor with series reactor and the shunt filters whose tuning frequency is below the $h^{\text {th }}$ harmonic, is to provide damping. To design a satisfied filter in the worst case, the effect of these devices can be neglected in the $h^{\text {th }}$ harmonic filter design.

In contrast, those shunt filters with tuning frequency above the $h^{\text {th }}$ harmonic, present a capacitive susceptance at the $h^{\text {th }}$ harmonic, and therefore increase the $h^{\text {th }}$ harmonic current flowing into the supply system due to the parallel resonance with system inductance [4].

Considering the amplifying effect of those higher order harmonic filters, the $h^{\text {th }}$ harmonic current injected into the supply line at the installation of $h^{\text {th }}$ harmonic filter is given by

$$
I_{S h}=\left|\frac{1}{1+Z_{S h} \cdot Y_{F}}\right| \cdot I_{h}
$$

where

$$
\begin{aligned}
& Z_{S h} \approx j X_{S h}=j \omega_{h} L_{S} \\
& Y_{F}=\sum_{i \geq h} \frac{1}{Z_{F i}} \\
& Z_{F i}=R+j \omega_{h} L_{i}+1 / j \omega_{h} C_{i}
\end{aligned}
$$

The design objective of an $h^{\text {th }}$ STF is to reduce the $h^{\text {th }}$ supply harmonic current $I_{S h}$ to an allowable level $I_{S h \text {.lim, which is }}$ recommended in [1].

\section{Optimization of Tuning Frequency}

Searching the optimal tuning frequency, to have the filter satisfy harmonic control indices with minimum capacity or with a given capacity, features largely in this approach.

Let $\omega_{d}$ denotes the desired tuning frequency of the $h^{\text {th }}$ harmonic filter, the partial resonant ratio $\varepsilon$ is defined as

$$
\varepsilon=\left(\omega_{h}-\omega_{d}\right) / \omega_{d}
$$

That means, the filter is tuned to

$$
n=h /(1+\varepsilon)
$$

\subsection{Optimal Tuning Frequency for Minimum Capacity}

A filter with minimum capacity is one that adequately suppresses harmonics at the minimum capacity of filter capacitor, regardless of the reactive power compensation. A well chosen $\varepsilon$ should have the filter satisfy its requirements at the worst point of maximum negative detuning $\delta_{n m}$ and positive detuning $\delta_{p m}$. If $\varepsilon$ is subject to the following constraint, the filter capacity will be minimized:

$$
\left.I_{S h}\right|_{\delta=\varepsilon+\delta_{p m}}=\left.I_{S h}\right|_{\delta=\varepsilon+\delta_{n m}}=I_{S h \text { max }}
$$

In this optimization problem, the system formulations can be generalized for obtaining the optimal values of $\varepsilon$ and $R$ by minimizing the error

$$
\begin{aligned}
& \min _{\varepsilon, R}\left\{\left|I_{S h}\right|_{\delta=\varepsilon+\delta_{p m}}-\left.I_{S h \text { max }}|+| I_{S h}\right|_{\delta=\varepsilon+\delta_{n m}}-I_{S h \text { max }} \mid\right\} \\
& \text { S.T. } \quad \varepsilon \in\left[-\delta_{p m},-\delta_{n m}\right], \quad R \in\left[0, X_{S h}\right]
\end{aligned}
$$

And then the inductance and capacitance can be obtained by

$$
\begin{aligned}
& L_{h}=q R_{h}(1+\varepsilon) / \omega_{h} \\
& C_{h}=(1+\varepsilon)^{2} /\left(\omega_{h}^{2} L_{h}\right)
\end{aligned}
$$

Where, $\mathrm{q}$ denotes the quality factor of the filter. Take a $5^{\text {th }}$ single tuned filter design as an example. Provided that the maximum deviation of system frequency, filtering capacitance, and inductance are $\pm 1 \%, \pm 2 \%$ and $[-8 \%,+2 \%]$ respectively, then $\delta_{\mathrm{pm}}=+3 \%$ and $\delta_{\mathrm{nm}}=-6 \%$. At the case of $\mathrm{q}=40$ and $I_{\text {Sh.lim }}^{*}=0.1$, the minimum capacity requirement of the filter at the optimal tuning frequency is compared with that at zero partial ratio and the experienced partial ratio, as listed in tab.1. Obviously, the minimum capacity of the filter is achieved at the optimal tuning frequency.

Tab.1 Design results for the final $5^{\text {th }}$ harmonic filter

\begin{tabular}{cccc}
\hline $\begin{array}{c}\text { Filter } \\
\text { Parameters }\end{array}$ & $\begin{array}{c}\text { Null } \\
\text { partiality }\end{array}$ & $\begin{array}{c}\text { Experienced } \\
\text { partiality }\end{array}$ & $\begin{array}{c}\text { Optimal } \\
\text { partiality }\end{array}$ \\
\hline$\varepsilon$ & 0.0 & 6.0 & 2.0 \\
$\mathrm{n}$ & 5.0 & 4.7 & 4.9 \\
$R_{\mathrm{h} \cdot \max }^{*}$ & 0.019 & 0.015 & 0.027 \\
$\left(\omega_{d} L_{\mathrm{h}}\right)^{*}$ max & 0.74 & 0.61 & 1.08 \\
$\left(\omega_{d} C_{\mathrm{h}}\right)^{*}{ }_{\min }^{*}$ & 1.35 & 1.64 & 0.93 \\
capacity $^{*}$ & 1.00 & 1.21 & 0.68 \\
\hline
\end{tabular}




\subsection{Optimal Tuning Frequency at Given Capacity}

Shunt filter is also a reactive power compensator at fundamental frequency. If the filters designed previously for the minimum capacity is not enough to meet the load's demand for reactive power, the capacity of one or more harmonic filters needs to be increased. In this case, the filter capacitance is determined by the reactive power to be compensated, and the resistance and inductance can be described by the following expression

$$
\begin{aligned}
& R_{h}=(1+\varepsilon) /\left(q \omega_{h} C_{h}\right) \\
& L_{h}=(1+\varepsilon)^{2} /\left(\omega_{h}^{2} C_{h}\right)
\end{aligned}
$$

Where, $\varepsilon$ can be obtained by minimizing either of the following errors

$$
\begin{gathered}
\min _{\varepsilon}\left\{\left|I_{S h}\right|_{\delta=\varepsilon+\delta_{p m}}-I_{S h \cdot \max } \mid\right\} \\
\min _{\varepsilon}\left\{\left|I_{S h}\right|_{\delta=\varepsilon+\delta_{p m}}-\left.\boldsymbol{I}_{S h}\right|_{\delta=\varepsilon+\delta_{n m}} \mid\right\}
\end{gathered}
$$

The $\varepsilon$ getting from (7) will slightly less than that from (8). Applying (7) will make the filter more safety, but (8) will make the filter more efficient.

\section{Design and Simulation}

According to the optimizing method and the system model mentioned above, a PCbased filter design procedure is implemented as shown in Fig.3.

Simulation program can be used, before and after the filter design, to analyze the distribution system for the behavior of harmonic currents and voltages as function of time, and to determine harmonic currents and the bus voltage distortion. Simulation is also intended to verify the filter design and check the effectiveness of the filter on different operating conditions, as well as the reduction of the filter effectiveness due to minor harmonics neglected in the process of filter design [3]. Fig.4 shows a MATLAB-based simulation code for the analysis of industrial power system with harmonic filters.

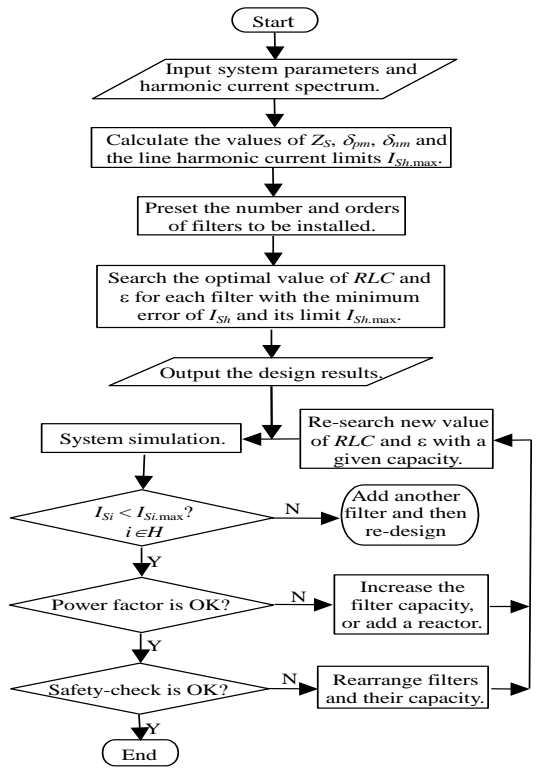

Fig.3 Decision flow chart for ST filter design

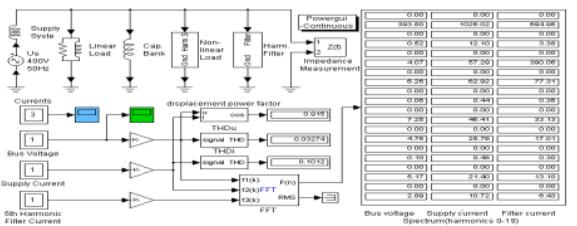

Fig.4 Simulation code for effect analysis of filter

For the sake of safety and reliability, simulation is also necessary for the designer to check the overload of capacitors upon the worst conditions. The capacitors must be able to withstand the shunting harmonics in addition to the normal fundamental quantities. The overload factors of power, voltage and current on the capacitor should not exceed their limits, otherwise the capacitor ratings should be modified.

To test the proposed method and the computer code, an industrial distribution system with a sintering furnace powered by a six-pulse phase-controlled converter was used for investigation. Fig. 1 shows the one-line diagram, and Tab.2 lists the system parameters and Tab.3 lists the 
current spectrum measured in the sintering furnace.

Tab.2 System parameters

\begin{tabular}{ll}
\hline \multicolumn{1}{c}{ Parameters } & Value \\
\hline Short circuit capacity at PCC & $90 \mathrm{MVA}$ \\
Transformer rating & $1.25 \mathrm{MVA}$ \\
Transformer impedance(pu) & 0.05 \\
Distribution bus voltage & $400 \mathrm{~V} / 50 \mathrm{~Hz}$ \\
Maximum load / current $\mathrm{I}_{\mathrm{L}}$ & $1 \mathrm{MVA} / 1443 \mathrm{~A}$ \\
Power in linear load & $0.4 \mathrm{MW}+\mathrm{j} 0.2 \mathrm{Mvar}$ \\
\hline
\end{tabular}

Tab. 3 Harmonic currents in sintering furnace

\begin{tabular}{|c|c|c|c|c|c|c|c|}
\hline & \multicolumn{2}{|c|}{ Current } & \multirow{2}{*}{ Phase } & \multirow{2}{*}{$\mathrm{h}$} & \multicolumn{2}{|c|}{ Current } & \multirow{2}{*}{ Phase } \\
\hline & (A) & $(\%)$ & & & (A) & $(\%)$ & \\
\hline 1 & 808 & $56.0 \%$ & $-65^{\circ}$ & 11 & 81 & $5.6 \%$ & $-175^{\circ}$ \\
\hline 3 & 9 & $0.6 \%$ & $165^{\circ}$ & 13 & 44 & $3.0 \%$ & $55^{\circ}$ \\
\hline 5 & 340 & $23.6 \%$ & $-145^{\circ}$ & 15 & 0.8 & $0.1 \%$ & $105^{\circ}$ \\
\hline 7 & 141 & $9.8 \%$ & $-95^{\circ}$ & 17 & 36 & $2.5 \%$ & $-25^{\circ}$ \\
\hline 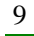 & 0.8 & $0.1 \%$ & $135^{\circ}$ & 19 & 18 & $1.2 \%$ & $-155^{\circ}$ \\
\hline
\end{tabular}

Tab.4 Design results for minimum capacity

\begin{tabular}{cccrrr}
\hline Filter & $n$ & \multicolumn{1}{c}{$R$} & \multicolumn{1}{c}{$L$} & \multicolumn{1}{c}{$C$} & \multicolumn{1}{c}{$V A R$} \\
\hline $5^{\text {th }}$ & 4.84 & $3.2 \mathrm{~m} \Omega$ & $65 \mathrm{uH}$ & $6760 \mathrm{uF}$ & $355 \mathrm{kvar}$ \\
$7^{\text {th }}$ & 6.60 & $19.8 \mathrm{~m} \Omega$ & $290 \mathrm{uH}$ & $810 \mathrm{uF}$ & $42 \mathrm{kvar}$ \\
\hline
\end{tabular}

Tab.5 The worst simulation result of primary design

\begin{tabular}{cccccc}
\hline Filter & \multicolumn{1}{c}{$I_{S 5}$} & $I_{S 7}$ & $T D D i$ & $T H D u$ & $\cos \phi_{1}$ \\
\hline None & $23.2 \%$ & $9.6 \%$ & $25.9 \%$ & $8.4 \%$ & 0.68 \\
$5^{\text {th }}+7^{\text {th }}$ & $7.0 \%$ & $6.4 \%$ & $10.4 \%$ & $4.0 \%$ & 0.91 \\
\hline
\end{tabular}

As shown in tab.5, the filters previously formed for minimum size are with some problems. The better solution is to use a $5^{\text {th }}$ single-tuned harmonic filter alone and enlarge its capacity to compensate the load reactive power. If necessary, the $5^{\text {th }}$ filter can be divided into 3 or more branches to adopt the variation of reactive power in different operating conditions.

Redesign the $5^{\text {th }}$ harmonic filter with the given capacity of 500kvar, and other parameters remain the same as the primary design. The design result is in Tab.6, and the simulation results are listed in Tab.7. Obviously the filter based on (7) has a smaller over-current factor $\left(K_{O C}\right)$ and the filter based on $(8)$ is relatively more efficient.
Tab.6 Design results for the final 5th harmonic filter

\begin{tabular}{ccccc}
\hline Optimizing Eq. & $n$ & $R$ & $L$ & $C$ \\
\hline Eq.7 & 4.67 & $2.4 \mathrm{~m} \Omega$ & $49 \mathrm{uH}$ & $9500 \mathrm{uF}$ \\
Eq.8 & 4.82 & $2.3 \mathrm{~m} \Omega$ & $46 \mathrm{uH}$ & $9500 \mathrm{uF}$
\end{tabular}

Tab.7 The worst simulation results of final design

\begin{tabular}{ccccccc}
\hline Eq. & $I_{S 5}$ & $I_{S 7}$ & $T D D i$ & $T H D u$ & $\cos \phi_{1}$ & $K_{O C}$ \\
\hline Eq.7 & $6.7 \%$ & $5.1 \%$ & $9.5 \%$ & $3.7 \%$ & 0.97 & 1.05 \\
Eq.8 & $5.3 \%$ & $4.9 \%$ & $8.3 \%$ & $3.4 \%$ & 0.97 & 1.06 \\
\hline
\end{tabular}

\section{Conclusion}

A PC-based design procedure for a single-tuned filter with an optimal tuning frequency is presented and a simulation program is accompanied to verify the effectiveness of the filter. Simulation results show that: (a) both methods satisfy the harmonic control indices; (b) design method can meet the requirement of power factor improving.

\section{Acknowledgment}

This work was supported by the Research foundation for the doctoral discipline project of higher education, China (20116118110006).

\section{References}

[1] IEEE Recommended Practices and Requirements for Harmonic Control in Electrical Power Systems, IEEE Std 519-1992.

[2] A.A. Abou El-Ela, S. Allam, H. ElArwash. "An optimal design of single tuned filter in distribution systems", Electric Power Systems Research, vol.78, pp.967-974, June 2008.

[3] J.M. Maza-Ortega, J.C.Churio Barboza, and M. Burgos-Payan "A software-based tool for optimal design of passive tuned filters", 2010 IEEE International Symposium on Industrial Electronics, 2010, pp.32733278.

[4] J.C. Das. "Passive filterspotentialities and limitations," IEEE Trans. Ind. Applicat., vol. 40, pp.232241, Jan./Feb. 2004. 